Meta

Journal des tradlucteurs

Translators' Journal

\title{
Par les détours de la robinetterie
}

\section{Claude Bédard}

Volume 23, numéro 4, décembre 1978

URI : https://id.erudit.org/iderudit/002016ar

DOI : https://doi.org/10.7202/002016ar

Aller au sommaire du numéro

Éditeur(s)

Les Presses de l'Université de Montréal

ISSN

0026-0452 (imprimé)

1492-1421 (numérique)

Découvrir la revue

Citer cet article

Bédard, C. (1978). Par les détours de la robinetterie. Meta, 23(4), 308-311.

https://doi.org/10.7202/002016ar d'utilisation que vous pouvez consulter en ligne.

https://apropos.erudit.org/fr/usagers/politique-dutilisation/ 


\section{PAR LES DÉTOURS DE LA ROBINETTERIE}

Tout traducteur technique que son travail amène à toucher au domaine de la robinetterie a le sentiment de nager dans une certaine confusion (qu'il attribuera de bonne grâce à sa relative ignorance en la matière) : de quelle manière, selon quelle logique employer tous ces équivalents de l'omniprésent générique anglais valve que les dictionnaires techniques bilingues servent aux sauces les plus variées et (refrain connu)... sans un mot d'explication? C'est sur cette question que j'ai voulu faire la lumière dans le présent article.

La première chose que l'on constate, après consultation d'un certain nombre d'ouvrages techniques, c'est que la confusion ressentie par le traducteur est pour le moins partiellement excusable : il y a en effet dans l'usage des termes de robinetterie un manque grave d'homogénéité. Heureusement, une planche de salut s'offre au chercheur: les normes AFNOR. Ces normes, sur lesquelles se sont alignés un certain nombre d'auteurs et de fabricants, ont le grand mérite de systématiser la nomenclature de la robinetterie; aussi constituent-elles la base de ma recherche. Cependant, elles ne résolvent pas toutes les difficultés et, par ailleurs, se heurtent parfois à des usages répandus et solidement enracinés dont il serait déraisonnable de ne pas tenir compte. Dans ces cas, je me suis permis de formuler des prises de position personnelles qui serviront, je l'espère, à faire avancer le débat.

Et tout d'abord, qu'est-ce donc qu'un appareil de robinetterie? Bien que sur ce point, déjà, l'unanimité ne soit pas complètel, on peut dire avec assez de certitude que ce terme désigne un dispositif installé sur une canalisation, un récipient, etc., contenant un fluide (liquide ou gaz) pour permettre, régler ou interrompre la circulation du fluide et dont l'organe essentiel est un obturateur. Cette définition exclut donc, soit qu'ils sont dépourvus d'obturateur, soit que celui-ci ne constitue pas leur organe essentiel:

$1^{\circ}$ Les séparateurs, filtres, purgeurs, injecteurs, éjecteurs et indicateurs, appelés appareils accessoires de tuyauterie ${ }^{2}$;

$2^{\circ}$ Les coudes, siphons, raccords, lyres ou joints de dilatation, éléments de tuyauterie tout à fait "passifs".

L'AFNOR divise les appareils de robinetterie en trois catégories générales que nous allons maintenant examiner en détail.

\section{A. LES ROBINETS}

Leur caractéristique essentielle - et exclusive - est que leur obturateur est commandé de l'extérieur. Cette commande peut être directe et volontaire (exemple: nos robinets domestiques, dits de puisage) ; elle peut aussi - c'est souvent le cas en robinetterie industrielle - être à distance (par l'intermédiaire d'une chaîne, d'une tringlerie, etc.) et asservie (exemple: un robinet actionné par un moteur électrique lui-même commandé par la pression interne d'un réservoir). Autre remarque importante: un robinet reste un robinet, quelle que soit sa taille.

Il existe une grande variété de robinets. La classification proposée par l'AFNOR a pour critère unique le type d'obturateur.

1. Certaines sources - peu nombreuses - étendent la définition de robinetterie à de simples éléments de tuyauterie: raccords, bondes, siphons, etc.

2. Cette expression, tirée de I'Encyclopédie des techniques industrielles Quillet, me semble nettement préférable (le «s" du dernier mot étant toutefois superflu) à la périphrase appareils accessoires d'installations pour tuyauterie, proposée par l'AFNOR. 
$1^{\circ}$ LE ROBINET À VANNE (fréquemment - et plus commodément - appelé robinet-vanne ) - L'obturateur, appelé vanne, effectue un mouvement de guillotine par rapport à la veine liquide; pour les besoins de la classification, on dit qu'il se déplace parallèlement à son siège (ou, plus précisément, à ses sièges, entre lesquels il coulisse).

Fait à noter, c'est là le seul sens que l'AFNOR accorde en robinetterie au mot vanne. Celui-ci, jusqu’alors aussi couramment employé que mal défini, pouvait désigner dans l'usage un robinet quelconque, plus spécialement un robinet-vanne, et était également associé aux robinets d'une "certaine» taille 3 .

$2^{\circ}$ LE ROBINET À SOUPAPE - L'obturateur, appelé soupape, a en général la forme d'un disque et se déplace perpendiculairement à son siège ${ }^{4}$. Ici, le terme proposé par l'AFNOR se heurte à un usage bien enraciné robinet à clapet. Le problème se complique davantage du fait qu'il n'existe pas de distinction précise entre les mots «soupape " et "clapet " (pour désigner un obturateur), que ceux-ci désignent doncà toutes fins pratiques la mêtme chose et qu'au surplus, l'usage favorise à cet égard le mot clapet ${ }^{5}$. L'utilisation indistincte de l'un ou de l'autre de ces termes n'entraînant pas d'ambiguité par ailleurs. j'estime que le choix reste ouvert sur ce point (voir la note 7).

Signalons aussi une variante importante du robinet à soupape: le robinet à pointeau. L'obturateur de ce robinet est essentiellement l'extrémité de la tige de manœuvre qui est usinée en forme de cône plus ou moins aigu (pour un cône très aigu, on utilise le mot aiguille) de façon à permettre un réglage très progressif de l'écoulement.

$3^{\circ}$ LE ROBINET À TOURNANT - Cette expression remplace l'ancien usage robinet $\grave{a}$ boisseau. L'obturateur, appelé tournant, se déplace par rotation dans un corps appelé boisseau; il affecte la forme d'un cylindre légèrement conique percé de lumières qui, lors de la manœuvre, masquent ou démasquent les tubulures du boisseau. Ce type de robinet (dont le nombre et la disposition des lumières et des tubulures varient largement) est apte à établir plusieurs circuits différents; c'est pourquoi il trouve une application privilégiée comme organe de sélection.

$4^{\circ}$ LE ROBINET À PAPILLON - L'obturateur, appelé papillon, est un disque plat et plus ou moins elliptique qui se déplace par pivotement sur un axe transversal (il suffit de se représenter le papillon d'un carburateur). Sur la désignation de ce robinet, tout le monde est et reste d'accord.

$5^{\circ}$ LE ROBINET Ȧ PAROI (OU Ȧ OBTURATEUR) DÉFORMABLE - Cee type de robinet, d'utilisation assez récente, n'est pas cité dans la classification AFNOR (qui date de 1946). Son principe est celui d'un étranglement dont l'une des parois latérales, élastique, se déplace de manière à accentuer l'étranglement jusqu'à le fermer, ou, dans l'autre sens, de manière à Pouvrir complètement. Dans ce cas, l'obturateur est appelé membrane. D'autre part, lorsque les deux parois latérales sont mobiles, l'obturateur qu'elles forment est appelé manchon.

3. Bien entendu, nul ne pouvait préciser à partir de quelle taille un robinet "devenait" une vanne... Aussi, bien que la connotation de grosseur soit encore répandue, j'estime que l'emploi du mot vanne n'apporte guère que de la confusion et qu'il faut s'habituer à employer le mot robinet dans tous les cas.

4. Notons au passage que le mot siège, confusion regrettable, désigne tant la surface de contact de l'obturateur que celle du corps de l'appareil. Ona heureusement la ressource d'éviter cette a mbiguité en utilisant le mot portée dans le premier cas et en réservant le mot siège au second.

5. Dans deux sources distinctes, il est étonnant de constater que l'auteur, ayant adopté le terme robinet à soupape, désigne l'obturateur par le mot clapet dans la description de l'appareil. 
$6^{\circ}$ LE ROBINET À PISTON - L 'obturateur, appelé piston, coulisse dans un cylindre percé d'un nombre variable d'orifices. À l'instar du robinet à tournant, ce robinet convient particulièrement aux circuits de commande hydraulique et pneumatique.

Il existe essentiellement deux constructions de piston: soit un cylindre creux ou plein percé de lumières transversales, soit une tige à laquelle sont fixées un certain nombre de portées en forme de disque. Cette dernière construction, fréquemment utilisée, a reçu de l'usage deux appellations vivaces: tiroir et valve. Ici encore, la confusion règne: tantôt les deux termes sont employés indistinctement, tantôt valve semble désigner un tiroir automatique actionné par les pressions antagonistes d'un fluide et d'un ressort (ou de deux fluides). Il s'agit en fait non plus d'un robinet dans ce cas, mais d'une soupape; certains utilisent d'ailleurs ce terme.

Le terme tiroir 6 , bien établi dans plusieurs domaines (machines à vapeur, commande hydraulique), me semble préférable à robinet à piston. Quant au terme valve, autrefois d'usage fréquent en robinetterie, il est en passe de devenir un anglicisme de maintien et c'est à ce titre, semble-t-il, qu'il a été complètement exclu par l'AFNOR; j'estime pour ma part qu'on peut s'en passer et utiliser à sa place les termes tiroir et soupape. Son seul usage indiscuté est celui de "valve de pneumatique " (il s'agit dans ce cas, à proprement parler, d'un clapet de non-retour), encore qu'il ne semble pas être fautif de l'utiliser dans le contexte de la commande hydraulique, où il conserve encore droit de cité.

\section{B. LES CLAPETS DE RETENUE}

Il s'agit d'appareils automatiques dont la fonction essentielle est de se fermer dans certaines conditions:

$1^{\circ}$ Soit l'inversion de l'écoulement dans la canalisation; on parle alors d'un clapet de non retour?

$2^{\circ}$ Soit encore un écoulement trop rapide dans la canalisation; il s'agit alors d'un clapet d'arrêt. Ce genre de dispositif est utilisé le plus souvent pour intervenir en cas de rupture de la canalisation.

6. Il existe peu de renseignements précis sur la terminologie du tiroir: aussi, je me permets de proposer ici les termes suivants: tiroir pour l'appareil lui-même, piston pour l'obturateur et portées pour les disques solidaires de la tige.

7. Cette orthographe est celle de l'AFNOR. Ailleurs, dans la plupart des cas, on ecrit non-retour. On trouve aussi l'expression clapet anti-retour.

8. Il semble y avoir lieu ici de critiquer la nomenclature de l'AFNOR. Nous avons vu que l'obturateur du robinet est (entre autres) la soupape, que celui du clapet est le clapet et que celui de la soupape est la soupape. $\vec{A}$ mon avis, cette terminologie introduit une double confusion:

$1^{\circ}$ Le même obturateur (du point de vue fonctionnement, forme, etc.) est ainsi. désigné, selon l'appareil, par des tcrmes différcnts.

$2^{\circ}$ Lorsqu'on considère un appareil en particulier, un seul et même terme désigne à la fois la partie et le tout, ce qui constitue une source très réelle d’ambiguités.

Voici, pour mieux illustrer mon propos, une proposition de solution "fictive ":

$1^{\circ}$ Utiliser le terme clapet, lorsqu'il s'agit d'un organe se déplaçant perpendiculairement à son siège pour désigner l'obturateur des trois appareils; si l'obturateur est d'un autre type, adopter le terme descriptif approprié (battant, piston, bille, etc.).

$2^{\circ}$ Désigner tant les appareils de non-retour que les appareils de surpression ou de dépression par le terme soupape. 11 existe en effet plus d'analogies que de différences entre ces appareils; ils sont notamment par opposition aux robinets, tous commandés par l'action du fluide. Il n'y aurait guère danger d’ambiguîté, par ailleurs, étant donné que l'usage fait normalement suivre le nom de ces appareils d'un complément indiquant leur fonction (anti-retour, de décharge, etc.).

Dans la pratique, il n'y aurait pas d'inconvénient à accepter le premier point; pour le deuxième, cependant, ce serait aller à l'encontre d'un usage trop bien établi. 
L'obturateur d'un clapet de retenue, quelle que soit sa nature, est appelé clapet. Il peut être articulé (on peut alors le désigner par le substantif battant), guidé (dans un déplacement perpendiculaire à son siège) ou encore complètement libre: il s'agit alors d'une bille ou boule (selon la grosseur).

\section{LES SOUPAPES AUTOMATIQUES}

Cette dernière catégorie englobe les appareils automatiques dont la caractéristique essentielle est de s'ouvrir lorsque la pression (ou la dépression) à l'intérieur de la canalisation ou du récipient atteint une valeur déterminée. L'obturateur, quelle que soit sa nature, est appelé soupape ${ }^{8}$; il s'agit le plus souvent d'un disque maintenu sur son siège par un ressort ou un poids.

La désignation des soupapes dépend de leur fonction; comme leurs applications sont variées, la terminologie est abondante. Contentons-nous de décrire deux types fondamentaux:

$1^{\circ}$ La soupape de détente (ou d'échappement, de décharge, de surpression, d'évacuation, de sûreté, etc.) sert à évacuer tout excès de fluide en cas de surpression.

$2^{\circ}$ La soupape d'admission ou d'aspiration, à l'inverse, sert à limiter la dépression à l'intérieur d'une canalisation ou d'une enceinte. On l'appelle couramment reniflard, cassevide, soupape anti-vide, etc.

Au terme de cette étude portant essentiellement sur la construction des appareils de robinetterie, j'aimerais signaler qu'il existe toute une terminologie complémentaire concernant la fonction des appareils: soit qu'il s'agisse d'ajouter au substantif de base des compléments ou adjectifs (clapet de pied, soupape de sécurité, robinet sélecteur, etc.), soit encore de le remplacer par un substantif de fonction (mitigeur, détendeur, distributeur, etc.). L'étude de cette terminologie serait longue et compliquée et n'entre pas, du reste, dans le cadre de mon propos.

Comme on peut le voir, tout n'est pas résolu, et il s'en faut de beaucoup. D'ailleurs, dans quel domaine technique les usages terminologiques sont-ils tout à fait rigoureux et universels? Dans la pratique, c'est souvent au traducteur de prendre personnellement position en s'appuyant sur une connaissance aussi complète que possible des données du problème; ainsi, sa terminologie, même si elle ne plaît pas à tous (comment le pourraitelle?), sera en tout cas choisie et utilisée de façon cohérente. Telle est du moins la démarche qui a inspiré le présent exposé.

Claude Bédard 